\title{
Video restoration and enhancement: algorithms and applications
}

\author{
Ling Shao · Jorge E. Caviedes · Kai-Kuang Ma • \\ Erwin Bellers
}

Published online: 28 January 2011

(C) Springer-Verlag London Limited 2011

With the evolution of telecommunication and multimedia technologies, consumer devices, such as HDTV, smart phones, portable media players and PDAs, have become many people's favorite gadgets in their daily lives. Those devices always have the functionality of playing videos either downloaded or captured using the embedded camera. Due to the limited wireless bandwidth and the shortage of storage capacity, video content played on consumer devices is often spatially and temporally down-sampled, corrupted with noise, and highly compressed. To fulfill a pleasant viewing experience, video restoration and enhancement algorithms can be applied to improve the picture quality of those video materials. Video restoration and enhancement has been a popular research topic for many years. Many existing algorithms have good performance, but they may not be applied for video enhancement on consumer devices directly. Consumer devices have certain properties

\author{
L. Shao $(\varangle)$ \\ Department of Electronic and Electrical Engineering, \\ The University of Sheffield, Mappin Street, Sheffield S1 3JD, UK \\ e-mail: ling.shao@sheffield.ac.uk \\ J. E. Caviedes \\ Intel Corporation, 5000 W. Chandler Blvd, CH7-428, Chandler, \\ AZ 85226, USA \\ e-mail: jorge.e.caviedes@intel.com \\ K.-K. Ma \\ School of Electrical and Electronic Engineering, \\ Nanyang Technological University, Block S2, Nanyang Avenue, \\ Singapore, Singapore \\ e-mail: ekkma@ntu.edu.sg \\ E. Bellers \\ Trident Microsystems, Inc., 1170 Kifer Road, Sunnyvale, \\ CA 94086, USA \\ e-mail: erwin.bellers@tridentmicro.com
}

that require special treatment for the video restoration and enhancement techniques, e.g., the resolution of mobile displays is often quite low, the processing units are relatively less powerful, and the memory size is limited. Therefore, novel algorithms must be developed or classical methods must be adapted to suit this new scenario.

This special issue looks back at the classical topic of video restoration and enhancement from a new angle. The purpose of the special issue is twofold: (1) stimulating new research activities in the area of video signal processing and (2) emphasizing the importance of practical issues for implementing video processing algorithms. After careful reviews, eight high-quality papers were selected for publication in this issue.

To effectively do video restoration and enhancement, image quality assessment metrics are often used first. In the article "No-reference Measurement of Perceptually Significant Blurriness in Video Frames", Dardi et al. present a blur measurement method in video frames without using a reference ideal image. The estimation is performed first through a global and simple measure over the whole picture, then through a finer, local analysis of the sharpness of the objects borders. The subjective relevance of the scene content is accounted for in choosing the image parts where this local blurriness measurement is performed. In article "Image quality assessment based on S-CIELAB model", He et al. propose a new image quality assessment framework that is based on color perceptual model. The image quality is assessed by incorporating excellent color perceptual characteristics model with the geometrical distortion measurement. First, the reference and distorted images are transformed into SCIELAB color perceptual space, and the transformed images are evaluated by existing metric in three color perceptual channels. The fidelity factors of three channels are weighted to obtain the image quality. 
The article "Adding explicit content classification to nonlinear filters", authored by $\mathrm{Hu}$ and de Haan, investigates several categories of nonlinear filters: order statistics filters, hybrid filters, neural filters, and bilateral filters with different forms of content classification in various image processing applications including image de-blocking, noise reduction, and image interpolation. The article "Repairing Imperfect Video Enhancement Algorithms Using Classification-Based Trained Filters", authored by Shao et al., presents a solution for repairing low-quality video at the back-end of a video chain. The method is based on image content classification and least squares trained filters, which train on ideal images and images with certain degradations. Experimental results show that the repairing technique can improve the image quality of various video enhancement modules. In the article "Noise reduction in high dynamic range images", Min et al. propose a noise reduction method in generating high dynamic range (HDR) images using a set of low dynamic range (LDR) images with different exposures, where ghost artifacts are effectively removed by image registration and local motion information. In high sensitivity setting, motion information is used in generating a HDR image.

Super-resolution is a fast-growing area for improving the resolution and sharpness of low-resolution images and videos. In the article "A Survey on Super-resolution Imaging", Tian and Ma provide a comprehensive review of super-resolution image and video reconstruction methods developed in the literature and highlight the future research challenges. In the article "Single Image SuperResolution using Self-Examples and Texture Synthesis", Damkat looks at super-resolution from a different angle. $\mathrm{He}$ proposes an algorithm for single image super-resolution based on example-based super-resolution and examplebased texture synthesis. The algorithm does not use an additional example database as it uses self-examples to synthesize new detail and texture, assuming that images contain a sufficient amount of self-similarity. The texture synthesis component of the algorithm enables the re-synthesis of texture at the output resolution to achieve super-resolution.

With the popularity of 3DTV, in the article "Discontinuity-Based Registration of Depth and Video Data in Depth Image Based Rendering", Fieseler and Jiang present a depth image-based rendering (DIBR) method to create content for 3DTV. The authors show that by using an edge-based registration method, the spatial alignment of depth and video data can be improved, leading to an alleviation of the observed artifacts. 